\title{
ON THE THEORY OF COHERENT PAIR PRODUCTION IN CRYSTALS IN PRESENCE OF ACOUSTIC WAVES
}

\author{
A. R. Mkrtchyan, A. A. Saharian*, L. Sh. Grigoryan, B. V. Khachatryan \\ Institute of Applied Problems in Physics, \\ 25 Nersessian Str., 375014 Yerevan, Armenia
}

November 3, 2018

\begin{abstract}
The influence of hypersonic waves excited in a single crystal is investigated on the process of electron-positron pair creation by high-energy photons. The coherent part of the corresponding differential cross-section is derived as a function of the amplitude and wave number of the hypersound. The values of the parameters are specified for which the latter affects remarkably on the pair creation cross-section. It is shown that under certain conditions the presence of hypersonic waves can result in enhancement of the process cross-section.
\end{abstract}

\section{Introduction}

The fundamental aspects of the high-energy electromagnetic processes in condensed media have been under discussion for a long time. In crystals, where the atoms are arranged in ordered rows, the cross-sections of the high-energy electromagnetic processes can change essentially compared with the corresponding quantities for a single atom (see, for instance, 1, 2, 3, 国 and references therein). The cross-sections change because the kinematics of the processes dictate that the interactions be spread over a significant distance. The momentum transfer between a highly relativistic interacting particle and the crystal can be small, especially along the direction of particle motion. When this longitudinal momentum transfer is small, the uncertainty principle dictates that the interaction be spread out over a distance, known as the formation length for particle production or, more generally, as the coherence length. If the formation length exceeds the interatomic spacing the interference effects from all atoms within this length are important and they can essentially affect the corresponding cross-sections.

From the point of view of controlling the parameters of the high-energy electromagnetic processes in a medium it is of interest to investigate the influence of external fields, such as acoustic waves, temperature gradient etc., on the corresponding characteristics.

${ }^{*}$ E-mail: saharyan@server.physdep.r.am 
The considerations of concrete processes, such as diffraction radiation [5], transition radiation [6], parametric X-radiation [7], channeling radiation [8], have shown that the external fields can essentially change the angular - frequency characteristics of the radiation intensities. In the present paper we investigate the influence of a hypersonic wave on the coherent creation of electron-positron pairs by high-energy photons in a crystal (for the analogous influence in the case of bremsstrahlung see [9]). We specify the conditions under which the external deformation field changes the pair creation cross-section compared to the case of an undeformed crystal and demonstrate the possibility for the positron yield enhancement. Electron-positron pair creation phenomena by high-energy photons passing through a crystal are interesting not only from the viewpoint of underlying physics (for a recent review see [10]) but also from the viewpoint of practical applications for generation of intense positron beams. The basic source to creating positrons for high-energy electronpositron colliders is the electron-positron pair creation by hard bremsstrahlung photons produced when a powerful electron beam hits an amorphous target. Another approach is to use a crystal, the atomic rows of which are aligned with the incident electron beam, instead of an amorphous target. Due to the coherent bremsstrahlung this gives more intense radiation of photons, and as a result higher positron intensity. To further improve the positron yield the channeling radiation can be used, which is more intense than coherent bremsstrahlung and gives more electron-positron pairs [11, 12, 13, 14, 15, 16, 17, 18, 19]. An additional increase in the yield of positrons can be reached by using acoustic waves excited in crystals [20].

The paper is organized as follows. In the next section a formula is derived for the coherent part of the electron-positron pair creation by a photon in presence of the sinusoidal deformation field generated by a hypersound. The analysis of the general formula and numerical results in the special cases when the photon enters into the crystal at small angle with respect to crystallographic axes or planes is presented in Sec. 3. The main results are re-mentioned and discussed in Sec. 6 .

\section{Cross-section of the pair creation}

The differential cross-section for the electron-positron pair creation in a crystal by a highenergy photon with the wave vector $\mathbf{k}$ can be written in the form (see, for example, [1, 2])

$$
\sigma(\mathbf{q}) \equiv \frac{d^{4} \sigma}{d E_{+} d^{3} q}=\left|\sum_{n} e^{i \mathbf{q} \mathbf{r}_{n}}\right|^{2} \sigma_{0}(\mathbf{q}),
$$

with $\mathbf{r}_{n}$ being the positions of atoms in the crystal, $\sigma_{0}(\mathbf{q})$ is the corresponding cross-section on an individual atom as a function of momentum transfer to the crystal $\mathbf{q}=\mathbf{k}-\mathbf{p}_{+}-\mathbf{p}_{-}$. Here $\mathbf{p}_{+}, E_{+}$and $\mathbf{p}_{-}, E_{-}$are the momenta and energies for the craeted positron and electron respectively (hereafter the system of units $\hbar=c=1$ is used). The interference factor in Eq. (1) is responsible for coherent effects arising due to periodical arrangement

of the atoms in a crystal. After averaging on thermal fluctuations the cross-section takes the standard form [1]

$$
\sigma(\mathbf{q})=\left\{N_{0}\left(1-e^{-q^{2} \overline{u_{t}^{2}}}\right)+e^{-q^{2} \overline{u_{t}^{2}}}\left|\sum_{n} e^{i \mathbf{q r}_{n 0}}\right|^{2}\right\} \sigma_{0}(\mathbf{q}),
$$


where $\mathbf{r}_{n}=\mathbf{r}_{n 0}+\mathbf{u}_{t n}, \mathbf{u}_{t n}$ is the displacement of atoms with respect to the equilibrium positions $\mathbf{r}_{n 0}$ (by taking into account the crystal deformation due to the hypersonic wave) due to the thermal vibrations,$\overline{u_{t}^{2}}$ is the temperature dependent mean-squared amplitude of the thermal vibrations of atoms in the crystal, $N_{0}$ is number of atoms in the crystal, $e^{-q^{2} \overline{u_{t}^{2}}}$ is the Debye-Waller factor. When external influences are present the positions of atoms can be written as

$$
\mathbf{r}_{n 0}=\mathbf{r}_{n e}+\mathbf{u}_{n},
$$

with $\mathbf{r}_{n e}$ being the equilibrium positions of atoms in the situation without deformation, $\mathbf{u}_{n}$ are the displacements of atoms caused by the acoustic wave. We will consider deformations with the sinusoidal structure

$$
\mathbf{u}_{n}=\mathbf{u}_{0} \sin \left(\mathbf{k}_{s} \mathbf{r}_{n e}+\varphi_{0}\right)
$$

where $\mathbf{k}_{s}$ is the wave vector of the hypersonic wave. Note that the dependence of $\mathbf{u}_{n}$ on time (through the phase $\varphi_{0}$ ) we can disregard, as for particle energies we are interested in, the characteristic time for the change of deformation field is much greater than the passage time of particles through the crystal. For the deformation field given by Eq. (4) the sum over the atoms of a crystal in (2) can be transformed into the form

$$
\sum_{n} e^{i \mathbf{q} \mathbf{r}_{n 0}}=\sum_{m=-\infty}^{+\infty} J_{m}\left(\mathbf{q} \mathbf{u}_{0}\right) e^{i m \varphi_{0}} \sum_{n} e^{i \mathbf{q}_{m} \mathbf{r}_{n e}}, \quad \mathbf{q}_{m}=\mathbf{q}+m \mathbf{k}_{s},
$$

where $J_{m}(x)$ is the Bessel function. For a lattice with a complex cell the coordinates of the atoms can be written as

$$
\mathbf{r}_{n e}=\mathbf{R}_{n}+\rho_{j},
$$

with $\mathbf{R}_{n}$ being the positions of the atoms for one of primitive lattices, and $\rho_{j}$ are the equilibrium positions for other atoms inside $n$ - th elementary cell with respect to $\mathbf{R}_{n}$. Now the sum over atoms of the lattice can be presented as

$$
\sum_{n} e^{i \mathbf{q}_{m} \mathbf{r}_{n e}}=S\left(\mathbf{q}_{m}\right) \sum_{n} e^{i \mathbf{q}_{m} \mathbf{R}_{n}}, \quad S(\mathbf{q})=\sum_{j} e^{i \mathbf{q} \rho_{j}}
$$

where $S(\mathbf{q})$ is the structure factor. By making use of the formulae given above the square of the modulus for the sum (5) takes the form

$$
\left|\sum_{n} e^{i \mathbf{q r}_{n 0}}\right|^{2}=\sum_{m, m^{\prime}=-\infty}^{+\infty} J_{m}\left(\mathbf{q u} \mathbf{u}_{0}\right) J_{m^{\prime}}\left(\mathbf{q} \mathbf{u}_{0}\right) e^{i\left(m-m^{\prime}\right) \varphi_{0}} \sum_{n, n^{\prime}} e^{i \mathbf{q}_{m} \mathbf{R}_{n}} e^{-i \mathbf{q}_{m^{\prime}} \mathbf{R}_{n^{\prime}}} S\left(\mathbf{q}_{m}\right) S^{*}\left(\mathbf{q}_{m^{\prime}}\right) .
$$

For thick crystals the sum over cells can be presented as a sum over the reciprocal lattice:

$$
\sum_{n} e^{i \mathbf{q}_{m} \mathbf{R}_{n}}=\frac{(2 \pi)^{3}}{\Delta} \sum_{\mathbf{g}} \delta\left(\mathbf{q}_{m}-\mathbf{g}\right),
$$

where $\Delta$ is the unit cell volume, and $\mathbf{g}$ is the reciprocal lattice vector. By taking into account the $\delta$ - function the quantity $\mathbf{q}_{m^{\prime}}$ can be written as $\mathbf{q}_{m^{\prime}}=\mathbf{g}+\left(m^{\prime}-m\right) \mathbf{k}_{s}$. As for the lattice one has $e^{-i \mathbf{g} \mathbf{R}_{n}}=1$, we receive

$$
\sum_{n^{\prime}} e^{-i \mathbf{q}_{m^{\prime}} \mathbf{R}_{n^{\prime}}}=\sum_{n^{\prime}} e^{-i\left(m^{\prime}-m\right) \mathbf{k}_{s} \mathbf{R}_{n^{\prime}}}=\frac{(2 \pi)^{3}}{\Delta} \sum_{\mathbf{g}} \delta\left(\left(m^{\prime}-m\right) \mathbf{k}_{s}-\mathbf{g}\right) .
$$


Firstly let us show that in the sum over $m$ the main contribution comes from the terms for which $m \mathbf{k}_{s} \mathbf{u}_{0} \lesssim \mathbf{g u}_{0}$, or equivalently $m \lesssim \lambda_{s} / a$, where $\lambda_{s}=2 \pi / k_{s}$ is the wavelength of the external excitation, and $a$ is the lattice constant. Indeed, due to the $\delta$-function in (9) the Bessel function enters in the form $J_{m}\left(\mathbf{g} \mathbf{u}_{0}-m \mathbf{k}_{s} \mathbf{u}_{0}\right)$. For terms with $m \mathbf{k}_{s} \mathbf{u}_{0} \gg \mathbf{g} \mathbf{u}_{0}$, by taking into account that for practically important cases $u_{0} / \lambda_{s} \ll 1$, and making use of the asymptotic formula for the Bessel function, for large values of the order we can see that $J_{m}\left(m \mathbf{k}_{s} \mathbf{u}_{0}\right) \sim(2 \pi m)^{-1 / 2}\left(\pi e u_{0} / \lambda_{s}\right)^{m}, m \gg \lambda_{s} / a \gg 1$, and the contribution of these terms is exponentially suppressed. This conclusion is also valid for the sum over $m^{\prime}$. Now let us consider the contribution of the summands $m \neq m^{\prime}$ in the sum (8). It follows from formula (10) that $\left|m-m^{\prime}\right| \gtrsim \lambda_{s} / a$. As $\lambda_{s} / a \gg 1$, from here we see that one of the numbers $m, m^{\prime}$ is large. Let it is the number $m^{\prime}, m^{\prime} \gtrsim \lambda_{s} / a$. For the argument of the Bessel functions one has the estimate $\mathbf{q u}_{0} \sim \mathbf{g u}_{0} \sim 2 \pi u_{0} / a$, and, hence, the ratio of the Bessel function order to the argument is estimated as $m^{\prime} / \mathbf{q u} \mathbf{u}_{0} \sim \lambda_{s} / 2 \pi u_{0}$. As for the practically important cases $u_{0} / \lambda_{s} \ll 1$, for the function $J_{m^{\prime}}\left(\mathbf{q} \mathbf{u}_{0}\right)$ we have

$$
J_{m^{\prime}}\left(\mathbf{q u} \mathbf{u}_{0}\right) \sim \sqrt{\frac{a}{2 \pi \lambda_{s}}}\left(\frac{\pi e u_{0}}{\lambda_{s}}\right)^{\lambda_{s} / a}
$$

It follows from here that under the condition $u_{0} / \lambda_{s} \ll 1$ the contribution of the terms with $m \neq m^{\prime}$ in the sum (8) is small compared to the diagonal terms. In the case $m=m^{\prime}$ the sum in the left hand side of (10) is equal to the number of cells, $N$, in a crystal and the square of the modulus for the sum on the left of Eq.(8) can be written as

$$
\left|\sum_{n} e^{i \mathbf{q r}_{n 0}}\right|^{2}=N \frac{(2 \pi)^{3}}{\Delta} \sum_{m=-\infty}^{+\infty} J_{m}^{2}\left(\mathbf{q} \mathbf{u}_{0}\right)\left|S\left(\mathbf{q}_{m}\right)\right|^{2} \sum_{\mathbf{g}} \delta\left(\mathbf{q}_{m}-\mathbf{g}\right) .
$$

Note that in this case we have no dependence on the phase $\varphi_{0}$.

In formula (2) the first two terms in figure braces do not depend on the direction of the vector $\mathbf{q}$ and correspond to the contribution of incoherent effects. The third summand depends on the orientation of crystal axes with respect to the vector $\mathbf{q}$ and determines the contribution of coherent effects. The corresponding part of the cross-section is known as an interference term and, by taking into account the formula for $\sigma_{0}(\mathbf{q})$ (see, e.g., [1, 2]), can be written as

$$
\sigma_{c}=\frac{e^{2}}{(2 \pi)^{3} \omega^{2}} \frac{q_{\perp}^{2}}{q_{\|}^{2}}\left|u_{\mathbf{q}}\right|^{2}\left(\frac{\omega \delta}{m_{e}^{2}}-1+\frac{2 \delta}{q_{\|}}-\frac{2 \delta^{2}}{q_{\|}^{2}}\right) e^{-q^{2} \overline{u_{t}^{2}}}\left|\sum_{n} e^{i \mathbf{q} \mathbf{r}_{n 0}}\right|^{2},
$$

where $u_{\mathbf{q}}$ is the Fourier-transform of the atomic potential, $\mathbf{q}_{\|}$and $\mathbf{q}_{\perp}$ are the parallel and perpendicular components of the vector $\mathbf{q}$ with respect to the direction of the photon momentum $\mathbf{k}, \omega$ is the photon frequency, $\delta=1 / l_{c}$ is the minimum longitudinal momentum transfer, and $l_{c}=2 E_{+} E_{-} /\left(\omega m_{e}^{2}\right)$ is the formation length for the pair creation process. Usually one writes the quantity $u_{\mathbf{q}}$ as $4 \pi Z e^{2}[1-F(q)] / q^{2}$, where $Z$ is the number of electrons in an atom, and $F(q)$ is the atomic form-factor. For the exponential screening of the atomic potential one has $u_{\mathbf{q}}=4 \pi Z e^{2} /\left(q^{2}+R^{-2}\right)$, with $R$ being the screening radius.

The general expression for the pair creation cross-section can be presented in the form

$$
d \sigma=N_{0}\left(d \sigma_{n}+d \sigma_{c}\right),
$$


where $d \sigma_{n}$ and $d \sigma_{c}$ are the cross-sections for the non-coherent and coherent processes of the electron-positron pair creation in a crystal per single atom. Using formulae (12) and (13) and integrating over $\mathbf{q}$ the cross-section for the coherent part can be presented as

$$
\begin{aligned}
\frac{d \sigma_{c}}{d E_{+}}= & \frac{e^{2} N}{\omega^{2} N_{0} \Delta} \sum_{m, \mathbf{g}} \frac{g_{m \perp}^{2}}{g_{m \|}^{2}}\left[\frac{\omega^{2}}{2 E_{+} E_{-}}-1+2 \frac{\delta}{g_{m \|}}\left(1-\frac{\delta}{g_{m \|}}\right)\right]\left|u_{\mathbf{g}_{m}}\right|^{2} \times \\
& \times J_{m}^{2}\left(\mathbf{g}_{m} \mathbf{u}_{0}\right)|S(\mathbf{g})|^{2} e^{-g_{m}^{2} \overline{u_{t}^{2}}}, \quad \mathbf{g}_{m}=\mathbf{g}-m \mathbf{k}_{s}
\end{aligned}
$$

where the summation goes under the constraint

$$
g_{m \|} \geq \delta
$$

For the simplest crystal with one atom in the elementary cell one has $N=N_{0}$ and $S(\mathbf{g})=1$. Formula (15) differs from the corresponding formula for the creation of pairs in an undeformed crystal (corresponding to the summand with $m=0$ and $u_{0}=0$, see, for instance, [西, []) by replacement $\mathbf{g} \rightarrow \mathbf{g}_{m}$, and additional summation over $m$ with weights $J_{m}^{2}\left(\mathbf{g}_{m} \mathbf{u}_{0}\right)$. This corresponds to the presence of an additional one dimensional lattice with the reciprocal lattice vector $m \mathbf{k}_{s}, m=0, \pm 1, \pm 2, \ldots$. Note that by taking into account the $\delta$-function in Eq. (12) the momentum conservation law can be written down as

$$
\mathbf{k}=\mathbf{p}_{+}+\mathbf{p}_{-}+\mathbf{g}-m \mathbf{k}_{s}
$$

where $-m \mathbf{k}_{s}$ stands for the momentum transfer to the external field.

\section{Analysis of the general formula}

In formula (15) for the pair creation cross-section the main contribution comes from the terms with $g_{m \|} \sim \delta$. It follows from here that the external excitation with a wave vector $\mathbf{k}_{s}$ will influence on the process of pair creation if $m k_{s \|} \gtrsim \delta$. As a consequence of the well-known properties of the Bessel function, in the sum over $m$ the main contribution is due to the summands with

$$
m \lesssim \mathbf{g}_{m} \mathbf{u}_{0} \sim g u_{0} \sim \frac{2 \pi u_{0}}{a}
$$

From the last two relations it follows that it is necessary to take into account the influence of external fields on pair creation by photons if

$$
\frac{u_{0}}{\lambda_{s}} \gtrsim \frac{a}{(2 \pi)^{2} l_{c}} .
$$

It should be noted that at high energies $a / l_{c} \ll 1$ and condition (19) does not contradict to the condition $u_{0} / \lambda_{s} \ll 1$. In view of the expression for the formation length, condition (19) can be also written as

$$
\frac{u_{0}}{\lambda_{s}} \gtrsim \frac{a m_{e}}{8 \pi^{2}} \frac{m_{e} \omega}{E_{+} E_{-}} .
$$

As for the process of pair creation $\omega>E_{ \pm}$, this condition is stronger than in the case of bremsstrahlung (see [9]). 
Let us consider the case of the simplest crystal with one atom in the elementary cell assuming that the photon enters into the crystal at small angle $\theta$ with respect to the crystallographic axis $z$ of the orthogonal lattice. The corresponding reciprocal lattice vector components are $g_{i}=2 \pi n_{i} / a_{i}, n_{i}=0, \pm 1, \pm 2, \ldots$, where $a_{i}, i=1,2,3$ are the lattice constants in the corresponding directions. We can write

$$
g_{m \|}=g_{m z} \cos \theta+\left(g_{m y} \cos \alpha+g_{m x} \sin \alpha\right) \sin \theta
$$

where $\alpha$ is the angle between the projection of the vector $\mathbf{k}$ on the plane $(x, y)$ and axis $y$. For small angles $\theta$ the main contribution into the cross-section comes from the summands with $g_{z}=0$ and we receive

$$
\frac{d \sigma_{c}}{d E_{+}} \approx \frac{e^{2}}{\omega^{2} \Delta} \sum_{m, g_{x}, g_{y}} \frac{g_{\perp}^{2}}{g_{m \|}^{2}}\left[\frac{\omega^{2}}{2 E_{+} E_{-}}-1+2 \frac{\delta}{g_{m \|}}\left(1-\frac{\delta}{g_{m \|}}\right)\right]\left|u_{g_{m}}\right|^{2} J_{m}^{2}\left(\mathbf{g}_{m} \mathbf{u}_{0}\right),
$$

where $g_{\perp}^{2}=g_{x}^{2}+g_{y}^{2}$, and the summation goes over the region $g_{m \|} \geq \delta$ with

$$
g_{m \|} \approx-m k_{z}+\left(g_{y} \cos \alpha+g_{x} \sin \alpha\right) \theta .
$$

Note that in the argument of the Bessel function $\mathbf{g}_{m} \mathbf{u}_{0} \approx \mathbf{g}_{\perp} \mathbf{u}_{0}$. It follows from here that if the displacements of the atoms in the acoustic wave are parallel to the axis $z$ then the main contribution into the cross-section is due to the summand with $m=0$ and the influence of the acoustic wave is small. The most promising case is the transversal acoustic wave propagating along the $z$ - direction. If the photon moves far from the crystallographic plane (the angles $\alpha$ and $\pi / 2-\alpha$ are not small) the expression under the sum is a smooth function on $g_{x}$ and $g_{y}$, and the summation over these variables can be replaced by integration:

$$
\sum_{g_{x}, g_{y}} \rightarrow \frac{a_{1} a_{2}}{(2 \pi)^{2}} \int d g_{x} d g_{y}
$$

We now assume that the photon enters into the crystal at small angle $\theta$ with respect to the crystallographic axis $z$ and near the crystallographic plane $(y, z)$ ( $\alpha$ is small). In this case with an increase of $\delta$ some sets of terms in the sum will fall out. This can essentially change the cross-section. Two cases have to be distinguished. Under the condition $\delta \sim 2 \pi \theta / a_{2}$ in Eq. (22) for the longitudinal component one has

$$
g_{m \|} \approx-m k_{z}+\theta g_{y} \geq \delta
$$

This relation does not depend on the component $g_{x}$ and the summation over this component can be replaced by integration $\sum_{g_{x}} \rightarrow\left(2 \pi / a_{1}\right) \int d g_{x}$. When $\mathbf{u}_{0} \| \mathbf{a}_{1}$, for exponential screening the corresponding integral is expressed in terms of the hypergeometric functions. Here we will consider in detail the simpler case $\mathbf{u}_{0} \| \mathbf{a}_{2}$. For the exponential screening after the elementary integration over $g_{x}$ we obtain

$$
\frac{d \sigma_{c}}{d E_{+}} \approx \frac{4 \pi^{2} Z^{2} e^{4}}{\omega^{2} a_{2} a_{3}} \sum_{m, g_{y}} \frac{2 g_{y}^{2}+R^{-2}}{g_{m \|}^{2}\left(g_{y}^{2}+R^{-2}\right)^{3 / 2}}\left[\frac{\omega^{2}}{2 E_{+} E_{-}}-1+2 \frac{\delta}{g_{m \|}}\left(1-\frac{\delta}{g_{m \|}}\right)\right] J_{m}^{2}\left(g_{y} u_{0}\right),
$$

where the summation goes under the condition (25). 

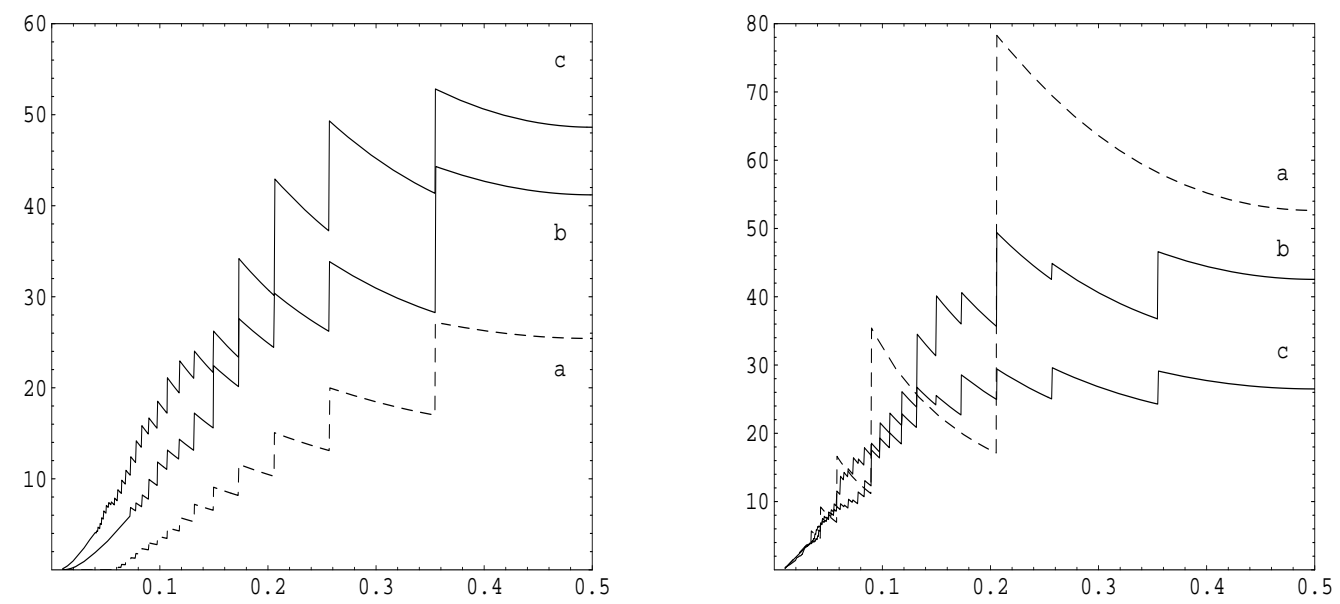

Figure 1: Pair creation cross-section, $\left(m_{e}^{2} \omega / Z^{2} e^{6}\right) d \sigma_{c} / d E_{+}$, as a function of $E_{+} / \omega$ for various values of the hypersound amplitude: $2 \pi u_{0} / a_{1}=0$ (a, dashed curves), 4 (b), 8 (c) and for $\psi=0.0005$ (left), $\psi=0.0035$ (right). The values for the other parameters are as follows: $\omega=100 \mathrm{GeV}, a_{1} / \lambda_{s}=5 \cdot 10^{-4}, a_{1} / 2 \pi R=1$.

Now we will assume that $\delta \sim 2 \pi \theta \alpha / a_{1}$. The main contribution into the sum in Eq. (22) is due to the terms with $g_{y}=0$ and we have two sums: over $m$ and $n_{1}, g_{x}=2 \pi n_{1} / a_{1}$. For the corresponding cross-section one receives:

$$
\frac{d \sigma_{c}}{d E_{+}} \approx \frac{e^{2}}{\omega^{2} \Delta} \sum_{m, n_{1}} \frac{g_{x}^{2}}{g_{m \|}^{2}}\left[\frac{\omega^{2}}{2 E_{+} E_{-}}-1+2 \frac{\delta}{g_{m \|}}\left(1-\frac{\delta}{g_{m \|}}\right)\right]\left|u_{g_{m}}\right|^{2} J_{m}^{2}\left(g_{x} u_{0 x}\right),
$$

where

$$
g_{m \|} \approx-m k_{z}+g_{x} \psi, \quad \psi=\alpha \theta,
$$

and summation goes over the values $m$ and $n_{1}$ satisfying the condition

$$
\left|n_{1} \psi-m a_{1} / \lambda_{s}\right| \geq \frac{m_{e}^{2} a_{1}}{4 \pi E_{+}\left(1-E_{+} / \omega\right)} .
$$

In this case the most favorable conditions to have an influence on the pair creation crosssection due to external fields are $\mathbf{u}_{0} \| \mathbf{a}_{1}$ (to have large values for $m$ ) and $\mathbf{k}_{s} \| \mathbf{a}_{3}$ (to have large values for $m k_{z}$ ). We have numerically evaluated the pair creation cross-section by making use of formula (27) for various values of parameters $\psi, u_{0}, \lambda_{s}$. The corresponding results show that, in dependence of these parameters, the external excitation can either enhance or reduce the cross-section. As an illustration in fig. I we have depicted the quantity $\left(m_{e}^{2} \omega / Z^{2} e^{6}\right) d \sigma_{c} / d E_{+}$as a function of $E_{+} / \omega$ in the case of cubic lattice $\left(a_{1}=\right.$ $a_{2}=a_{3}$ ) and exponential screening of the atomic potential for $u_{0}=0$ (dashed curves (a)), $2 \pi u_{0} / a_{1}=4$ (curves (b)), $2 \pi u_{0} / a_{1}=8$ (curves (c)) and for $\psi=0.0005$ (left), $\psi=0.0035$ (right). The values for the other parameters are as follows: $\omega=100 \mathrm{GeV}$, $a_{1} / \lambda_{s}=5 \cdot 10^{-4}, a_{1} / 2 \pi R=1$. As the cross-section is symmetric under the replacement $E_{+} / \omega \rightarrow 1-E_{+} / \omega$ we have plotted the graphs for the region $0 \leq E_{+} / \omega \leq 0.5$ only. In fig. 2 we have presented the cross section evaluated by Eq. (27) as a function of $2 \pi u_{0} / a_{1}$ for the positron energy corresponding to $E_{+} / \omega=0.4$ and for $\psi=0.0005$ (full curve), $\psi=0.0035$ (dashed curve). The values for the other parameters are the same as in fig. 11. 


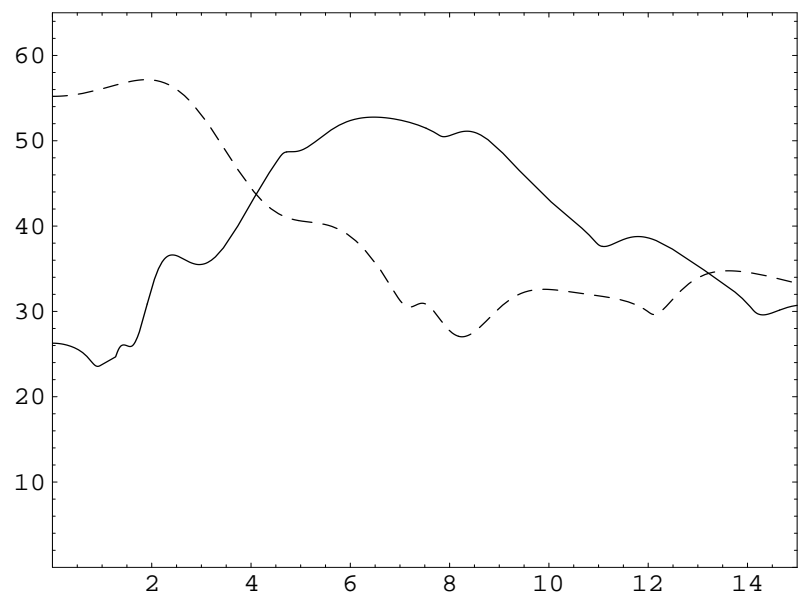

Figure 2: Pair creation cross-section, $\left(m_{e}^{2} \omega / Z^{2} e^{6}\right) d \sigma_{c} / d E_{+}$, as a function of $2 \pi u_{0} / a_{1}$ for the positron energy corresponding to $E_{+} / \omega=0.4$ and for $\psi=0.0005$ (full curve), $\psi=0.0035$ (dashed curve). The values for the other parameters are the same as in fig. 11.

\section{Conclusion}

In this paper we have investigated the electron-positron coherent pair creation by highenergy photons in a single crystal in the presence of a hypersonic wave. If the displacements of the atoms in the crystal under the influence of hypersound have the form (4), the coherent part of the corresponding cross-section per single atom, averaged on thermal fluctuations, is given by formula (15). To compared with the cross-section in an undeformed crystal this formula contains an additional summation over the reciprocal lattice vector $m \mathbf{k}_{s}$ of the one dimensional superlattice induced by the hypersonic wave. The contribution for a given $m$ is weighted by $J_{m}^{2}\left(\mathbf{g}_{m} \mathbf{u}_{0}\right)$, where the vector $\mathbf{g}_{m}$ is defined as in Eq. (15). We have substantiated that the influence of the hypersound on the cross-section can be remarkable under the condition (20). Note that for $u_{0} \gtrsim a$ this condition is less restrictive than the naively expected one $l_{c} \gtrsim \lambda_{s}$. In section 3 we have considered the most interesting case when the photon enters into the crystal at small angle with respect to a crystallographic axis (axis $z$ in our consideration). The main contribution into the coherent part of the cross-section comes from the crystallographic planes, parallel to the chosen axis. The behaviour of this cross-section as a function on the positron energy essentially depends on the angle between the projection of the photon momentum on the plane $(x, y)$ and a crystallographic plane. The corresponding numerical evaluations show that the presence of the hypersonic wave can either enhance or reduce the cross-section. This can be used to control the parameters of the positron sources for storage rings and colliders. Note that for the positron sources based on the channeling radiation the hypersound can also substantially influence on the corresponding intensity of the radiated photons (see Refs. [8]). 


\section{Acknowledgment}

The work has been supported by Grant no. 1361 from Ministry of Education and Science of the Republic of Armenia.

\section{References}

[1] M. L. Ter-Mikaelian, High Energy Electromagnetic Processes in Condensed Media (Wiley Interscience, New York, 1972).

[2] A. I. Akhiezer, N. F. Shulga, High Energy Electrodynamics in Matter (Gordon and Breach, Luxemburg, 1996).

[3] V. A. Bazylev, N. K. Zhevago, Radiation of Fast Particles in Matter and in External Fields (Nauka, Moscow, 1987) (in Russian).

[4] V. N. Baier, V. N. Katkov, V. N. Strakhovenko, High Energy Electromagnetic Processes in Aligned Single Crystals (Nauka, Novosibirsk, 1989) (in Russian).

[5] A. R. Mkrtchyan, L. Sh. Grigoryan, A. N. Didenko, A. A. Saharian, A. G. Mkrtchyan, Izv. Akad. Nauk. Arm. SSR. Fizika 24, 62 (1989); A. R. Mkrtchyan, L. Sh. Grigoryan, A. N. Didenko, A. A. Saharian, Sov. Phys. JTP 61, 21 (1991); A. R. Mkrtchyan, L. Sh. Grigoryan, A. A. Saharian, A. N. Didenko, Acustica 75, 1984 (1991); A. A. Saharian, A. R. Mkrtchyan, L. A. Gevorgian, L. Sh. Grigoryan, B. V. Khachatryan, Nucl. Instr. and Meth. B173, 211 (2001).

[6] L. Sh. Grigoryan, A. H. Mkrtchyan, A. A. Saharian, Nucl. Instr. and Meth. B145 197 (1998).

[7] A. R. Mkrthyan, H. A. Aslanyan, A. H. Mkrtchyan, R. A. Gasparyan, Phys. Lett. A152, 297 (1991).

[8] A. R. Mkrtchyan, R. A. Gasparyan, R. G. Gabrielyan, Phys. Lett. A115, 410 (1986); JETP 93432 (1987); Phys. Lett. A126, 528 (1988); L. Sh. Grigoryan et al., Rad. Eff. and Def. in Solids 152, 13 (2000); 152, 225 (2000); 152, 269 (2000); 153, 221 (2001); 153, 289 (2001); 153, 307 (2001); L. Sh. Grigoryan et al., Nucl. Instr. and Meth. B173, 13 (2001); B173, 184 (2001); L. Sh. Grigoryan, A. H. Mkrtchyan, H. F. Khachatryan, V. U. Tonoyan, W. Wagner, Nucl. Instr. and Meth. (2002) in press.

[9] A. A. Saharian, A. R. Mkrtchyan, L. A. Gevorgian, L. Sh. Grigoryan, B. V. Khachatryan, Influence of external excitations on bremsstrahlung in crystals, to be published.

[10] Yu. P. Kunashenko, D.Sc. Thesis (Tomsk, 2000) (in Russian).

[11] R. Chehab et al., Orsay Report LAL-RT 89-01, 1989.

[12] F. J. Decker, SLAC-PUB-5482,1991.

[13] X. Artru, V. N. Baier, R. Chehab, A. Jejcic, Nucl. Instr. and Meth. A344, 443 (1994). 
[14] X. Artru et al., Nucl. Instr. and Meth. B119, 246 (1996).

[15] K. Yoshida et al., Phys. Rev. Lett. 80, 1440 (1998).

[16] B. N. Kalinin et al.,Nucl. Instr. and Meth. B145, 209 (1998).

[17] V. N. Baier et al., Nucl. Instr. and Meth. B145, 221 (1998).

[18] M. Inoue et al., Nucl. Instr. and Meth. B73, 104 (2001).

[19] CERN Courier 41, No. 10, 10 (2001).

[20] W. Wagner, E. B. Uggerhoj, A. R. Mkrtchyan, R. O. Avakyan, P. A. Alexandrov, INTAS project application (1999). 